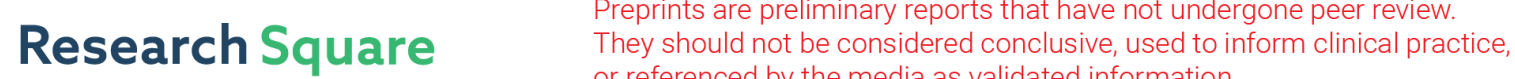 or referenced by the media as validated information. \\ Development of "NEC-SD-LAMP," a New Non- Electrified Infectious Diseases Testing Method
}

\section{Yusuke Kimura}

Tokyo Medical and Dental University

Masashi lkeuchi ( $\square$ ikeuchi.mech@tmd.ac.jp )

Tokyo Medical and Dental University

\section{Article}

Keywords: NEC-SD-LAMP, Infectious Diseases, Testing Method, SARS-CoV-2 infection

Posted Date: January 7th, 2022

DOI: https://doi.org/10.21203/rs.3.rs-1197771/v1

License: (c) (1) This work is licensed under a Creative Commons Attribution 4.0 International License. Read Full License 


\section{Abstract}

Here, non-electric-control SalivaDirect loop-mediated isothermal amplification (NEC-SD-LAMP), which can detect infections by analyzing viral gene expression in saliva without electrical control systems, was developed. In this method, viral genes are purified from saliva using SalivaDirect, and gene expression is analyzed by loop-mediated isothermal amplification (LAMP) by adding water to the device, and the results can be visually confirmed. Melting palmitic acid maintains the optimal temperature for the LAMP reaction, as the temperature of palmitic acid is maintained at $62.9^{\circ} \mathrm{C}$, its melting point. By taking advantage of the proximity of this melting point to the optimal temperature for LAMP, it is possible to perform LAMP without electricity. Using this method, adenovirus DNA was detected in saliva.

Furthermore, the detection limit was 2 copies per $\mu \mathrm{L}$, indicating that it is possible to detect viral infections in saliva even before the onset of SARS-CoV-2 infection.

\section{Introduction}

Infectious diseases are a major cause of death in developing countries ${ }^{1,2}$. One reason is the lack of diagnostic techniques. There are fewer hospital facilities in developing countries than in developed countries, limiting the number of facilities available for testing and treatment and greatly reducing the opportunities for testing; this is particularly important for viral infections. The early detection and treatment of viral infections are critical to treating and preventing the spread of infection. In addition, many tropical areas where neglected tropical diseases (NTDs) are prevalent and diagnostic techniques are required have neither electrical infrastructure nor stable voltage ${ }^{3}$. This makes it impossible to set up conventional testing devices that require electrical control. Therefore, to supply testing technology to developing countries, it is essential to establish methods that satisfy the following three criteria: 1) the entire process from testing to confirmation of results can be performed without using electrical control systems, 2) accurate detection of infectious diseases from the early stage, and 3) portability that enables testing anywhere such as in residential areas, outdoors, etc. [point of care testing (POCT)].

Several devices or methods can be used to perform infectious disease tests in non-electrified environments. First, there are several infection test kits based on antigen-antibody reactions such as immunochromatography ${ }^{4,5}$. By adding samples to the device, the infectious disease test can be performed quickly, and the results can be observed visually or using a smartphone. Although these devices can easily perform tests, they are prone to non-specific reactions and have low accuracy. In addition, it is difficult to detect infections in the early stages of disease because of the protein-detection method employed by these devices. Nucleic acid expression analysis is more effective for detecting infectious diseases because it enables early diagnosis by amplification and quantification.

Recombinase polymerase amplification (RPA) is an isothermal amplification method for nucleic acid analysis that can be performed in a non-electrified environment ${ }^{6,7}$. Using this method, the target gene can be amplified and detected by heating samples at $37^{\circ} \mathrm{C}$. Because of the low reaction temperature, this method can be performed using human body temperature. In addition, because this is a nucleic acid 
amplification method, it is possible to detect infectious diseases in their early stages. However, this method is prone to non-specific amplification reactions, and when used for infectious disease testing, many false positives occur. In addition, it is difficult to observe the results visually; therefore, it is necessary to use conventional analyzing devices that require electrical control, implying that the entire system cannot be applied in a non-electrified field.

The loop-mediated isothermal amplification (LAMP) method is an isothermal gene amplification method in which results can be visually observed ${ }^{8-10}$. This method amplifies and detects the target gene by heating the sample to a constant temperature of $60-65^{\circ} \mathrm{C}$. The LAMP method uses four or six primers in the amplification reaction, which improves the specificity of the target gene compared with that of PCR. When a target gene is amplified by LAMP, a substantial amount of pyrophosphate ions is produced. These bind to manganese ions with a quenching function in the calcein-manganese ion complex in the LAMP sample, dissociating them from calcein. As a result, calcein fluorescence increases as the reaction progresses; this phenomenon allows visual observation of target gene amplification ${ }^{11}$.

To perform LAMP in a non-electrified field, disposable heat packs have been used ${ }^{12}$. The LAMP method can be easily performed by placing the LAMP sample between two disposable heat packs, and the results can be visually confirmed. In addition, portability is improved. However, the output temperature of this method is unstable because the reaction is carried out using heat from two disposable heat packs, which are considerably affected by the surrounding environmental temperature-this especially affects tropical regions. Furthermore, the process of purifying the target gene from the specimen is not considered. Thus, the purification process must be performed differently. As a result, no method or device satisfies all three aforementioned criteria.

In this study, we developed a new practical method, "Non-Electric-Control SalivaDirect LAMP (NEC-SDLAMP)," which can purify viral genes from saliva, quantify the purified viral genes, and confirm the results visually by only adding water. This method can purify viral genes from saliva using the SalivaDirect method, amplify viral genes using the LAMP method, and confirm viral infection with the naked eye. The SalivaDirect method has been used to test for SARS-CoV-2 infections. In this method, viral proteins in saliva are denatured by adding proteinase $\mathrm{K}$. Through this process, viral genes are eluted and purified. At the same time, DNase and RNase in the saliva are inactivated so that the viral genes in the sample can be stably preserved. After the reaction, the sample is heated at $95^{\circ} \mathrm{C}$ for 5 min to inactivate proteinase $\mathrm{K}$, and the reaction is stopped ${ }^{13}$. With these simple operations, it is possible to purify viral genes in saliva.

NEC-SD-LAMP uses an exothermic agent and palmitic acid to perform the SalivaDirect and LAMP reactions, respectively, in a reaction device comprising an insulated container. Because the reaction is carried out in an insulated container, it can be conducted independently of the surrounding environmental temperature. When water is added to the NEC-SD-LAMP device, the exothermic agent within the device reacts and heats it internally to $95^{\circ} \mathrm{C}$. This process stops the SalivaDirect reaction. The heat produced by the exothermic agent is also used to melt palmitic acid, whose melting point is $62.9^{\circ} \mathrm{C}$. During the 
transition of palmitic acid from liquid to solid, the temperature is fixed at the melting point. By taking advantage of the proximity of this melting point to the optimal temperature, LAMP can be performed.

Based on these principles, we succeeded in heating at approximately $95^{\circ} \mathrm{C}$ for more than 5 min, which is necessary for proteinase $\mathrm{K}$ inactivation, and at $60-65^{\circ} \mathrm{C}$ for more than $1 \mathrm{~h}$, which is necessary for LAMP. This device is small $(20 \times 16 \times 14 \mathrm{~cm})$ and can be fabricated for less than $\$ 20$ USD. In addition, all components except for the exothermic agent and the sample tube in which the specimen comes into contact can be used semi-permanently. Palmitic acid is a fatty acid found in animals and plants; therefore, it is safe for use. Given the above, the device is low-cost and portable and can be used for POCT in developing countries, including tropical regions. When we used this device to detect adenovirus DNA in saliva, we detected a viral concentration of up to 2 copies per $\mu \mathrm{L}$. This is lower than the viral concentration in saliva at onset in patients with SARS-CoV-2 infection, indicating that early diagnosis of infectious diseases is possible using this device ${ }^{14}$.

\section{Results}

\section{Overview of NEC-SD-LAMP device}

Figure 1a shows the fabricated NEC-SD-LAMP device. The device consists of a "stand" to place the PCR tube with reagents when the SalivaDirect reaction is performed, an "exothermic agent," a "polypropylene container (PP container)" containing palmitic acid, a "vacuum insulated container (VI container)" used during the LAMP reaction, a "frame" to determine the position of these components, and a "polystyrene container (PS container)" to hold all the components. The same stand is placed inside the PP container, and the stand and frame were fabricated using a 3D printer. The stand has multiple holes for PCR tube placement, enabling the simultaneous reaction of multiple samples in one experiment (Fig. 1b). Furthermore, the stand is removable, and different stands with different numbers or sizes of holes can be used. By using other stages, we can change the number of samples in an experiment or perform experiments on tubes of other volumes. The exothermic agent comprises calcium oxide and aluminum. The PP container is made of a 200- $\mu \mathrm{m}$-thick PP film and fitted inside the VI container (Fig. 1c). Because it is made of a thin film, the PP container has high thermal conductivity, enabling efficient melting of palmitic acid during the exothermic reaction described below. The PS container is formed by combining $2.0-\mathrm{cm}$-thick polystyrene boards. The lid of the PS container has a $\varphi 1.0-\mathrm{cm}$ hole, which serves to release the hydrogen and water vapor generated during the SalivaDirect reaction to the outside of the container. When performing the SalivaDirect reaction, the PS container is closed and secured using a band, and a 2.0-mm-thick chloroprene rubber is mounted between the lid and container to seal the container.

Figure 2 shows the reaction process occurring in the developed device. During the SalivaDirect reaction, the stand, exothermic agent, and PP and VI containers (without lid) are placed in a PS container (Fig. 2a). First, proteinase $\mathrm{K}$ is added to the saliva sample in the PCR tube, and then the PCR tube is placed on a stand to perform the SalivaDirect reaction. After the reaction, MilliQ water is added to the PS container, 
and the lid of the PS container is closed, initiating the following reaction between MilliQ water and the exothermic agent in the PS container:

$\mathrm{CaO}+\mathrm{H}_{2} \mathrm{O}=\mathrm{CaOH}_{2}+15.2 \mathrm{Kcal} \ldots$.

$\mathrm{Al}+1 / 2 \mathrm{Ca}(\mathrm{OH})_{2}+2 \mathrm{H}_{2} \mathrm{O}=1 / 2 \mathrm{CaO}-\mathrm{Al}_{2} \mathrm{O}_{3}+3 / 2 \mathrm{H}_{2}+93.8 \mathrm{Kcal} \ldots(2)$

The heat generated by this reaction is used to 1) stop the SalivaDirect reaction through proteinase $\mathrm{K}$ inactivation, 2) melt palmitic acid in the PP vessel, and 3) heat the inside of the VI container.

After the SalivaDirect reaction, LAMP can be performed. When LAMP is performed, the PP container is placed in the VI container (Fig. 2b). The post-SalivaDirect sample is added to the LAMP reagent in a PCR tube and placed on a stand in the PP container. The lid of the VI container is then closed, and the LAMP reaction proceeds. The temperature in the PP container can be maintained at $62.9^{\circ} \mathrm{C}$, which is the melting point of palmitic acid, until all the palmitic acid in the PP container transitions from liquid to solid.

Because the reaction is performed in a $\mathrm{VI}$ container, it is not only possible to maintain the temperature of palmitic acid for a long time, but it is also possible to achieve a uniform reaction independent of the surrounding environment temperature. After the reaction, the solidified palmitic acid could be reused by removing the PP container from the VI container and reheating it. This dual-container structure allows for repeated use without changing any device components except for the heating agent. The above process enables overly sensitive analysis of viral infections without any electrical control systems.

\section{Temperature verification}

Figure 3a shows the temperature change inside the PS container during the exothermic agent reaction. In this experiment, $150 \mathrm{~mL}$ of water was added to the PS container, with the exothermic agent placed at the bottom. The temperature at each time point was calculated using the resistance of a thermistor set on the side of the PS container. As shown in Fig. 3a, this reaction was able to heat the container to $94.8^{\circ} \mathrm{C}$ for more than $5 \mathrm{~min}$. This is sufficient temperature and reaction time to inactivate proteinase $\mathrm{K}$. We also confirmed that all palmitic acid in the PP container could be melted by heating.

Figure $3 \mathrm{~b}$ shows the temperature change of the palmitic acid in the PP container. This experiment verified the temperature change in the melted palmitic acid after placing the PP container in the VI container after the exothermic reaction. The temperature at each time point was calculated using the resistance of a thermistor set on the side of the PP container. As shown in Fig. 3b, palmitic acid in the PP container was successfully maintained at $62.7^{\circ} \mathrm{C}$ for more than $1 \mathrm{~h}$. The temperature and reaction time were sufficient to perform LAMP.

\section{Viral gene detection experiment with NEC-SD-LAMP}

Figure 4 shows the results of a test to detect viral genes in saliva using the developed device. Figure $4 a$ shows the PCR tube after the reaction when NEC-SD-LAMP was performed using saliva samples mixed with adenovirus DNA. "Negative Control (NC)" referred to NEC-SD-LAMP performed using saliva samples 
without adenovirus DNA, while "Positive Control (PC)" referred to the SalivaDirect reaction and LAMP performed using saliva samples containing adenovirus DNA in a conventional thermal cycler, and "Sample" referred to NEC-SD-LAMP performed using saliva samples containing adenovirus DNA. As shown in Figure 4a, the solution color change of the sample was visually observed to be similar to that of the PC. At this time, it was possible to identify the sample under natural light without irradiating it with excitation light.

To prove that the SalivaDirect reaction was stopped by the exothermic reaction, NEC-SD-LAMP was performed using samples in which proteinase $\mathrm{K}$ was not inactivated by the exothermic agent. Gene amplification by LAMP did not occur even in samples containing adenovirus DNA (Sup. Fig. 1).

This result suggests that when proteinase $\mathrm{K}$ is not sufficiently inactivated, the enzyme in the LAMP reagent is inactivated, and the LAMP reaction is not performed correctly. Moreover, the results also suggest that proteinase $\mathrm{K}$ could be sufficiently inactivated by an exothermic reaction.

Supplementary Movie shows a video demonstration of NEC-SD-LAMP. As shown in Supplementary Movie, by just adding water and the samples, NEC-SD-LAMP can easily be used to purify genes from saliva, verify, and observe the results without any electrical control system.

\section{NEC-SD-LAMP in a tropical temperature environment}

The NEC-SD-LAMP device is especially designed for use in tropical regions, where infectious diseases are widespread. To verify whether a stable reaction can be performed even in tropical regions, we set up the device in an incubator $\left(50^{\circ} \mathrm{C}\right)$ and performed NEC-SD-LAMP. Saliva containing adenovirus DNA was used as the specimen and was successfully detected in the incubator reaction as well as at room temperature (Sup. Fig. 2). These results indicate that this system can perform stable reactions, even in hightemperature environments.

\section{Detection limit of NEC-SD-LAMP}

Figure $4 \mathrm{~b}$ shows the detection limit verification results of viral concentration when using NEC-SD-LAMP. Here, the PCR tube, after running NEC-SD-LAMP using saliva samples containing adenovirus DNA with final concentrations ranging from 0 to 200 copies per $\mu \mathrm{L}$, is shown. As shown in Figure $4 \mathrm{~b}$, samples containing more than 2 copies per $\mu \mathrm{L}$ of adenovirus DNA showed a change in solution color relative to the NC sample ( 0 copies per $\mu \mathrm{L}$ ), indicating that this method can detect samples containing more than 2 copies per $\mu \mathrm{L}$ of viral DNA.

We tested the possibility of performing NEC-SD-LAMP in a large sample volume using a 1.5-mL tube, assuming the detection of saliva samples with extremely low viral concentrations. The NEC-SD-LAMP device can be equipped with a 1.5-mL tube by changing the stand (Sup. Fig. 3a, b). In this experiment, we used a sample volume four times larger than that of the PCR tube. As a result, we were able to confirm a change in the solution color up to a virus concentration of 0.5 copy per $\mu \mathrm{L}$ (Sup. Fig. 3c), suggesting that NEC-SD-LAMP can be performed with only a change in the stand, even for large samples that require a 
large amount of heat to perform the reaction. It was also suggested that NEC-SD-LAMP could be used to detect viruses in saliva at very low concentrations.

\section{Discussion}

NEC-SD-LAMP can test viral infections in saliva samples without using any electrical controls by combining SalivaDirect and LAMP methods. Verification experiments were performed using adenoviral DNA. Owing to the characteristics of the LAMP method, it is possible to test for various viral infections by simply changing primer sequences.

Because the NEC-SD-LAMP system performs the reaction in highly insulated PS and VI containers, the reaction is not affected by high or low environmental temperatures and can be performed accurately. However, when conducting tests in tropical regions, it is necessary to bring not only the device but also the reagents to the field. Conventional reagents are difficult to use because they must be stored below $-20^{\circ} \mathrm{C}$. Nevertheless, lyophilized reagents that can be stored at room temperature have been developed ${ }^{15}$. Furthermore, dried reagents are already in practical use (Nippon Gene Co., Ltd., Tokyo, Japan; https://www.nippongenematerial.com/development/index_development.html). Therefore, combining the NEC-SD-LAMP system and dried reagents should be possible for field testing in tropical regions.

It was suggested that NEC-SD-LAMP could detect viral genes up to 2 copies per $\mu \mathrm{L}$. Since the viral concentration in patient saliva at the onset of SARS-CoV-2 is approximately $10-10^{4}$ copies per $\mu \mathrm{L}$, NECSD-LAMP can detect the virus in the early stage of infection ${ }^{14}$. Furthermore, in the $1.5-\mathrm{mL}$ tube reaction, the viral concentration could be detected up to 0.5 copy per $\mu \mathrm{L}$. Therefore, the NEC-SD-LAMP system can cope with future outbreaks of infectious diseases that occur even at lower virus concentrations.

The proposed system can perform the LAMP reaction by taking advantage of the fact that the melting point of palmitic acid is equal to the optimum temperature of LAMP. Other isothermal nucleic acid amplification methods include the smart amplification process (SmartAmp), RPA, and nucleic acid sequence-based amplification (NASBA) $)^{6,16,17}$. These methods can also be carried out in this system using substances with melting points similar to the optimum temperature for each reaction or paraffin adjusted to an appropriate melting point. Therefore, this system can be applied to various reaction types. However, because water vapor is generated during exothermic reactions, if a water-soluble substance is used for the isothermal amplification reaction, the reaction may not be performed accurately; therefore, it is preferable to use fat-soluble substances.

In the present study, we developed a novel method, "NEC-SD-LAMP," to detect infectious diseases by analyzing viral gene expression from saliva without using an electrical control system. In this method, the entire reaction can be carried out by adding MilliQ water to the device, and the method can be performed in a non-electrified environment, including observation of the results. In addition, it is a small device that allows the reuse of component parts, except for the exothermal agent, and the device can be fabricated for less than \$20 USD, making it possible to perform POCT at a low cost. Moreover, because the reaction 
is performed in an insulated container, it can be used in developing countries, including tropical regions, if dried reagents are used in combination. This device will make it possible to provide testing technology not only to developing countries but also to disaster areas where, for example, large-scale power outages occur. NEC-SD-LAMP is an innovative method that allows for easy testing of viral infections at any field site worldwide.

\section{Methods}

\section{Fabrication of NEC-SD-LAMP device}

Among the components of the NEC-SD-LAMP device, the PS container was fabricated by combining 2.0$\mathrm{cm}$-thick polystyrene boards. A $41.0-\mathrm{cm}$ diameter hole was opened in the lid to direct the generated water vapor and hydrogen outside the container. A 2.0-mm-thick chloroprene rubber was placed between the top of the container and lid, providing a seal between the lid and the container. Additionally, two bands were placed around the PS container to fix the lid to the container.

The stand and frame were fabricated using a Mojo 3D printer (Stratasys, Rehovot, Israel) with ABS resin (Mojo P430 QuickPack Print Engine; Stratasys) as the resin material.

The PP container was made from a 200- $\mu \mathrm{m}$-thick PP film. The aforementioned stand and $80 \mathrm{~g}$ of palmitic acid (Wako Pure Chemical Industries, Ltd., Osaka, Japan) were placed inside a container, and a handle made from the PP film was placed on top of the PP container for ease of operation.

An exothermic agent (Morians Heat Pack size L; Morian Heat Pack Co. Ltd., Irima City, Japan) and VI container (SR250; ASVEL, Yamatokoriyama-shi, Japan), which are commercially available products, were used.

\section{Reaction temperature measurement}

Temperature measurements during the exothermic agent reaction and the LAMP reaction with palmitic acid were carried out using a previously developed temperature control system ${ }^{18}$. Each temperature in the system was measured by measuring the resistance of a thermistor (104 JT; SEMITEC, Tokyo, Japan) at $50 \mathrm{~Hz}$ and running calculations on a computer. The resistance of the thermistor was inputted into a laptop using an analog I/O PC card (ADA16-8/2(CB)L; CONTEC Co., Ltd., Osaka, Japan), and the temperature calculation program was created using LabVIEW ver8.2 ( $N I$, Austin, TX, USA). During the exothermic reaction, the thermistor was placed on the side of the PS container. During the LAMP reaction, the thermistor was also placed on the side of the PP container after storage in the VI container such that it was in contact with the melted palmitic acid. The averages and deviations of the measured temperatures 3-13 and 3-63 min after commencing measurements during the exothermic and LAMP reactions, respectively, were calculated.

\section{NEC-SD-LAMP Verification Experiment}


The following experiments were conducted to verify the functionality of NEC-SD-LAMP.

$50 \mu \mathrm{L}$ of saliva from a healthy person were mixed with AMPLIRUN Adeno Virus DNA (MBC001; Vircell S.L., Grenada, Spain) to a final concentration of 100 copies per $\mu \mathrm{L}$ and added to the PCR tube. The SalivaDirect reaction was performed by mixing MagMAX ${ }^{\mathrm{TM}}$ Viral/Pathogen Proteinase K (Thermo Fisher Scientific, Waltham, MA, USA) with a saliva sample to a final concentration of $50 \mu \mathrm{g}$ per $\mu \mathrm{L}$ and incubating at room temperature for $5 \mathrm{~min}$. After the reaction, $30 \mu \mathrm{L}$ of mineral oil (M-5904; Sigma-Aldrich, St Louis, MO, USA) was added to prevent sample evaporation, and the PCR tube was placed on a stand. Thereafter, an exothermic agent, VI container without a lid, PP container, and stand with a PCR tube were placed inside the PS container. Subsequently, $150 \mathrm{~mL}$ of MilliQ water was added to react with the exothermic agent to heat the inside of the PS container to approximately $95^{\circ} \mathrm{C}$ for $5 \mathrm{~min}$ to inactivate the proteinase K. After the reaction, it was confirmed that all palmitic acid in the PP container had melted, after which it was placed in a VI container.

LAMP reagents were prepared by mixing a $2 \times$ reaction mix, enzyme mix, distilled water, fluorescence detection reagent (all from Eiken Chemical Co., Ltd., Tokyo, Japan), and six primers (FI, BI, F3, B3, Loop F, and Loop B; 001-00890; LAMP Primer Design and Synthesis Service; Nippon Gene, Tokyo, Japan) according to the RT-LAMP kit protocol (Eiken Chemical Co., Ltd.). Table.1 shows the primer sequences used to detect adenovirus DNA in this experiment. The final concentration of each primer was adjusted to $2 \mu \mathrm{M}$ (PI and BI primers), $0.25 \mu \mathrm{M}$ (F3 and B3 primers), and $1 \mu \mathrm{M}$ (Loop F and Loop B primers).

Thereafter, $20 \mu \mathrm{L}$ of the prepared LAMP reagent and $5 \mu \mathrm{L}$ of the post-SalivaDirect sample were added to the PCR tube (final concentration of adenovirus DNA: 20 copies per $\mu \mathrm{L}$ ). After adding $20 \mu \mathrm{L}$ of mineral oil to prevent evaporation, the PCR tube was placed on a stand in a PP container. LAMP was then performed by closing the lid of the $\mathrm{VI}$ container and placing it for $1 \mathrm{~h}$ on the laboratory table. After the reaction, the PCR tube was removed from the stand, and the color of the solution was observed under natural or excitation light using a SmartBlue Transilluminator (Greiner Bio-One, Frickenhausen, Germany), and each observation was captured using a camera (SO-03j; Sony Corporation, Tokyo, Japan).

Table 1. Primer sequences for detecting adenovirus DNA

\begin{tabular}{|c|c|}
\hline Primer & Sequence \\
\hline $\mathrm{F}$ & 5'-GCCTCCTTCGTGCGTGATGATATGGTTGGACGCTGG-3' \\
\hline $\mathrm{Bl}$ & 5'-AGTCGCGCAGCTTGTTGAATCAAGGAAACCCTGGACTA-3' \\
\hline F3 & 5'-GAAGACGATCTGCCTGAAG-3' \\
\hline B3 & 5'-AGGGACAGGATAAGTATGACAT-3' \\
\hline LoopF & 5'-CGGTAGGTCTTACAGACGC-3' \\
\hline LoopB & 5'-GGTGACCTGCACGTCTAG-3' \\
\hline
\end{tabular}

As a comparison experiment, the following four reactions were also performed.

As a negative control (NC), a saliva sample without AMPLIRUN adenovirus DNA was used, and NEC-SDLAMP was performed using the same reaction as above. 
As a positive control (PC), a saliva sample containing the same concentration of AMPLIRUN adenovirus DNA was prepared and heated at $95^{\circ} \mathrm{C}$ for 5 min to stop the SalivaDirect reaction and at $63^{\circ} \mathrm{C}$ for $1 \mathrm{~h}$ for the LAMP reaction using a T100 Thermal Cycler (Bio-Rad Laboratories, Hercules, CA, USA).

To verify the effect of the proteinase $\mathrm{K}$ residue, we prepared a sample that was not heated by the exothermic agent after the SalivaDirect reaction. LAMP with palmitic acid was used for this sample. The amount of sample and the concentration of reagents in each process were the same as above.

To verify the functionality of the NEC-SD-LAMP device in tropical regions, it was installed in an incubator (EO-450V; AS ONE Corporation, Osaka, Japan) heated to $50^{\circ} \mathrm{C}$, and NEC-SD-LAMP was performed. The amount of sample and the concentration of reagents in each process were the same as above.

\section{Verification of detection limit}

To verify the limit of detection concentration of NEC-SD-LAMP, the following experiments were performed.

$50 \mu \mathrm{L}$ of saliva from a healthy person were mixed with AMPLIRUN adenovirus DNA to a final concentration of 1,000 copies per $\mu \mathrm{L}$ and added to the PCR tube. The same procedure detailed in the "NEC-SD-LAMP Verification Experiment" was used to perform the SalivaDirect and exothermic agent reactions.

The LAMP method with palmitic acid heating was performed using the same procedure as in the "NECSD-LAMP Verification Experiment," after which the color of the solution was observed under natural or excitation light on a SmartBlue Transilluminator (Greiner Bio-One). For the LAMP method, the postSalivaDirect reaction sample was diluted using MilliQ water to final concentrations of $1,2,10,20,100$, and 200 copies per $\mu \mathrm{L}$ of adenovirus DNA.

As a comparison experiment, the following two reactions were also performed.

As a negative control (NC), a saliva sample without AMPLIRUN adenovirus DNA was used, and NEC-SDLAMP was performed using the same reaction as above (final concentration of adenovirus DNA: 0 copy per $\mu \mathrm{L})$.

As a large-volume NEC-SD-LAMP experiment, NEC-SD-LAMP was performed in a 1.5-mL tube. The reagent concentration was the same as described above, and the reaction was performed using a 4 -fold increase in the total solution volume. The final concentration of adenovirus DNA was adjusted to 0 copy per $\mu \mathrm{L}(\mathrm{NC}), 0.5$ copy per $\mu \mathrm{L}$, and 1 copy per $\mu \mathrm{L}$.

\section{Declarations}

\section{Data Availability Statement}

All data generated or analyzed during this study are included in this published article (and its Supplementary Information files). 
Acknowledgments

This work was supported by JST, ACT X, Japan, and Research Center for Biomedical Engineering.

\section{Author Contributions}

Y.K. and M.I. conceived the study. Y.K. performed the experiments and analyzed the data. M.I. contributed to the manuscript preparation, and Y.K. wrote the manuscript.

\section{Competing Interests}

The authors declare no competing interests.

\section{References}

1. Yager, P. et al. Microfluidic diagnostic technologies for global public health. Nature 442, 412-418 (2006).

2. Urdea, M.et al. Requirements for high impact diagnostics in the developing world. Nature 444, 73-79 (2006).

3. Flambard, J., Amirat, Y., Feld, G., Benbouzid, M. \& Ruiz, N. River and estuary current power overview. J. Mar. Sci. Eng. 7, 365 (2019).

4. Chen, Z. et al. Rapid and sensitive detection of anti-SARS-CoV-2 IgG, using lanthanide-doped nanoparticles-based lateral flow immunoassay. Anal. Chem. 92, 7226-7231 (2020).

5. Roda, A. et al. Dual lateral flow optical/chemiluminescence immunosensors for the rapid detection of salivary and serum IgA in patients with COVID-19 disease. Biosens. Bioelectron. 172, 112765; 10.1016/j.bios.2020.112765 (2021).

6. Piepenburg, O., Williams, C.H., Stemple, D.L. \& Armes, N.A. DNA detection using recombination proteins. PLOS Biology 4, e204; 10.1371/journal.pbio.0040204 (2006).

7. Kim, H. E. et al. Sensitive electrochemical biosensor combined with isothermal amplification for point-of-care COVID-19 tests, Biosens. Bioelectron. 182, 113168; 10.1016/j.bios.2021.113168 (2021).

8. Notomi, T. et al. Loop-mediated isothermal amplification of DNA. Nucleic Acids Res. 28, e63-e63 (2000).

9. Nagamine, K., Watanabe, K., Ohtsuka, K., Hase, T. \& Notomi. T. Loop-mediated isothermal amplification reaction using a nondenatured template. Clin. Chem. 47, 1742-1743 (2001).

10. Nagamine, K., Hase, T. \& Notomi, T. J. M. C. P. Accelerated reaction by loop-mediated isothermal amplification using loop primers, Mol. Cell Probes 16, 223-229 (2002).

11. Tomita, N., Mori, Y., Kanda, H. \& Notomi, T. Loop-mediated isothermal amplification (LAMP) of gene sequences and simple visual detection of products. Nat. Protoc. 3, 877-882 (2008).

12. Zhang, Y. et al. Point-of-care multiplexed assays of nucleic acids using microcapillary-based loopmediated isothermal amplification. Anal. Chem. 86, 7057-7062 (2014). 
13. Vogels, C. et al. SalivaDirect: RNA extraction-free SARS-CoV-2 diagnostics. protocols.io, dx.doi.org/10.17504/protocols.io.bjswknfe (2020).

14. Zhu, J., Guo, J., Xu, Y. \& Chen, X. Viral dynamics of SARS-CoV-2 in saliva from infected patients. J. Infect. 81, e48-e50 (2020).

15. Panno, S. et al. Loop mediated isothermal amplification: principles and applications in plant virology. Plants, 9, 461 (2020).

16. Mitani, Y. et al. Rapid SNP diagnostics using asymmetric isothermal amplification and a new mismatch-suppression technology. Nature Methods 3, 257-262 (2007).

17. Sharma, A. et al. COVID-19 Diagnosis: Current and Future Techniques. Int. J. Biol. Macromol. 21; 10.1016/j.jibiomac.2021.11.016 (2021).

18. Kimura, Y., Ikeuchi, M., Inoue, Y. and Ikuta, K. 3D microdevices that perform sample purification and multiplex qRT-PCR for early cancer detection with confirmation of specific RNAs. Sci. Rep. 8, 17480; 10.1038/s41598-018-35772-y (2018).

\section{Figures}


(a)

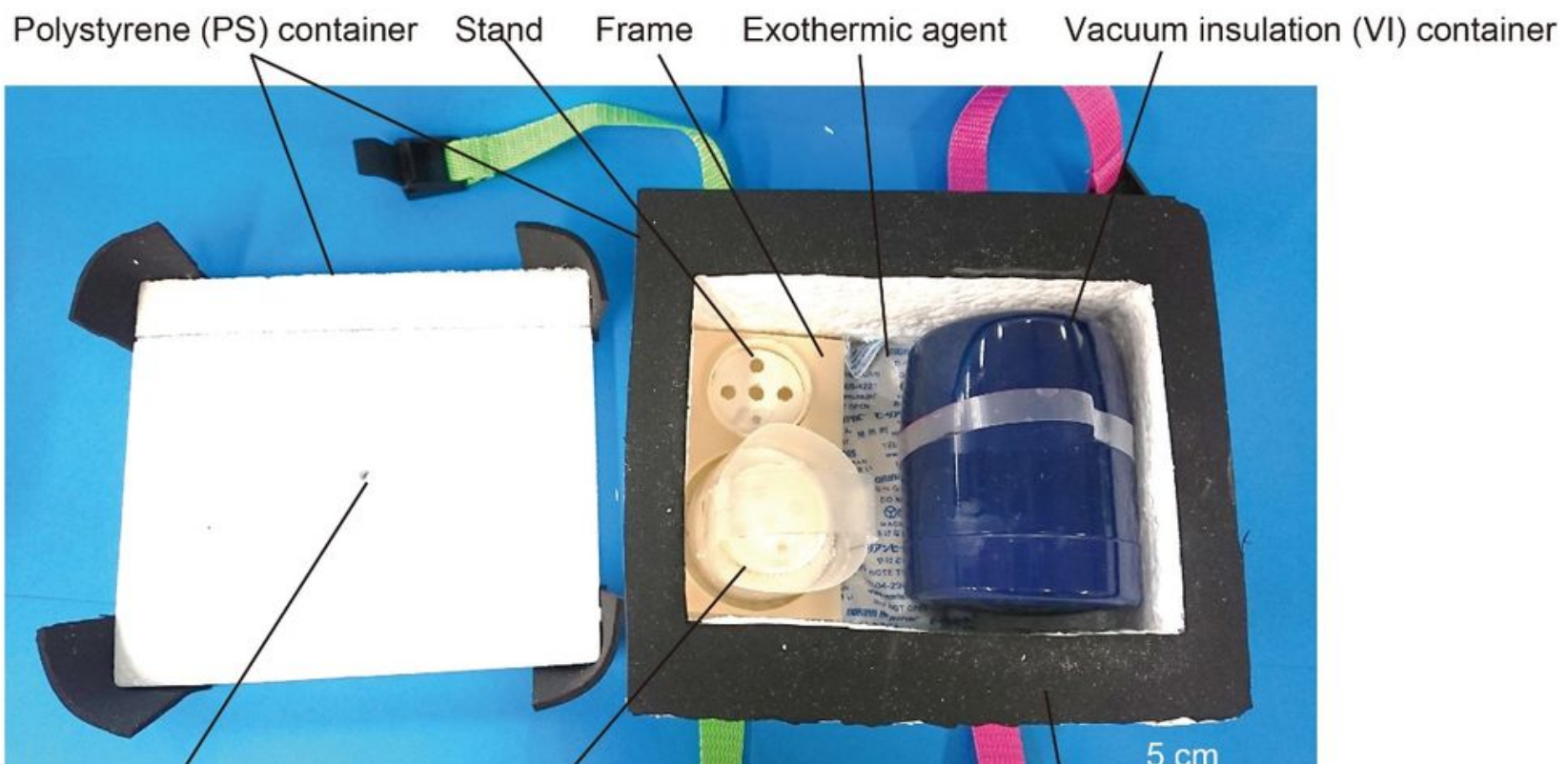

Figure 1

The developed NEC-SD-LAMP device. (a) All components of the NEC-SD-LAMP device. (b) Stand for the SalivaDirect assay. There are several holes on the stand to set the PCR tubes. (c) PP container. The stands for the PCR tube and palmitic acid are mounted inside the container. 
(a)

VI container

MilliQ (150 ml)

(Without a lid)
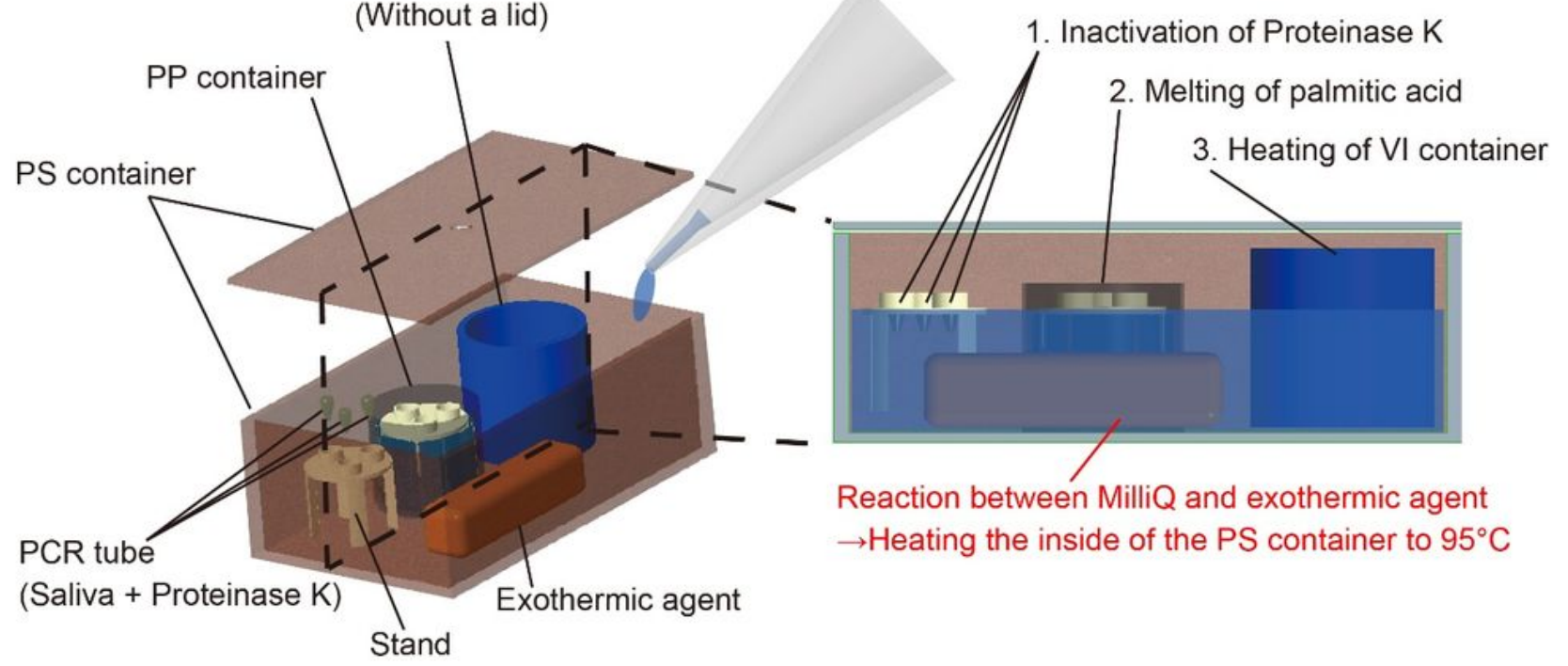

(b)

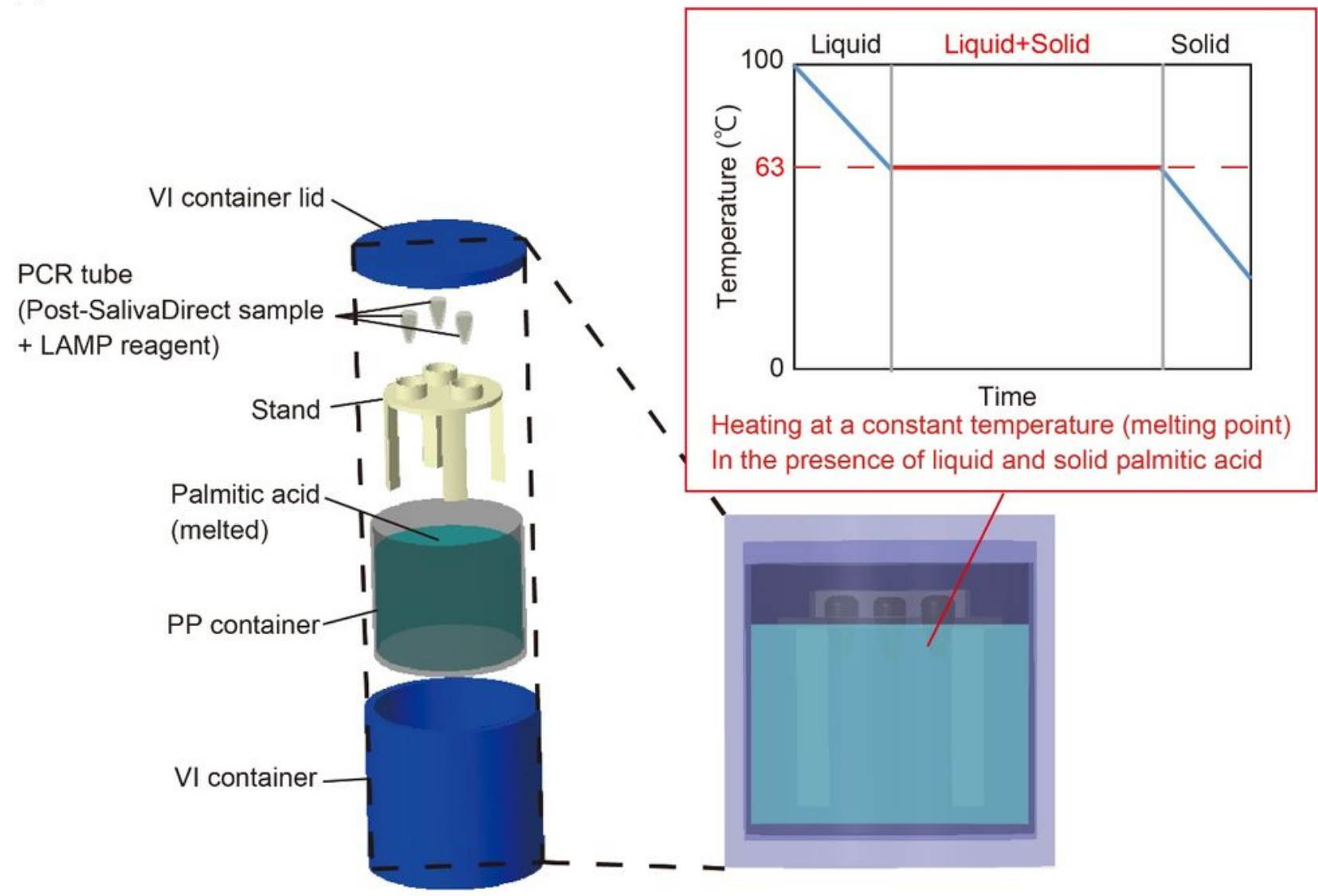

\section{Figure 2}

Mechanism of NEC-SD-LAMP. (a) Operation of the SalivaDirect assay. In this method, the exothermic reaction between MilliQ water and an exothermic agent is applied to stop the SalivaDirect reaction through proteinase $\mathrm{K}$ inactivation, dissolving palmitic acid in the PP container and heating the inside of the VI container. (b) Operation of the LAMP assay. During the execution of the LAMP assay, the PP container is placed inside the VI container, and the PCR tube containing the LAMP sample is placed on 
the stand of the PP container. The reaction is carried out by applying the transition heat of palmitic acid when it transitions from liquid to solid.

(a)

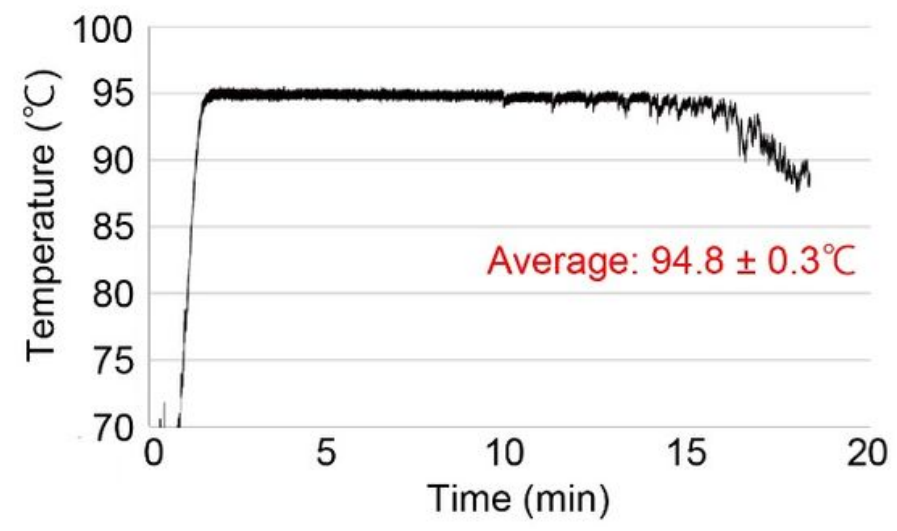

(b)

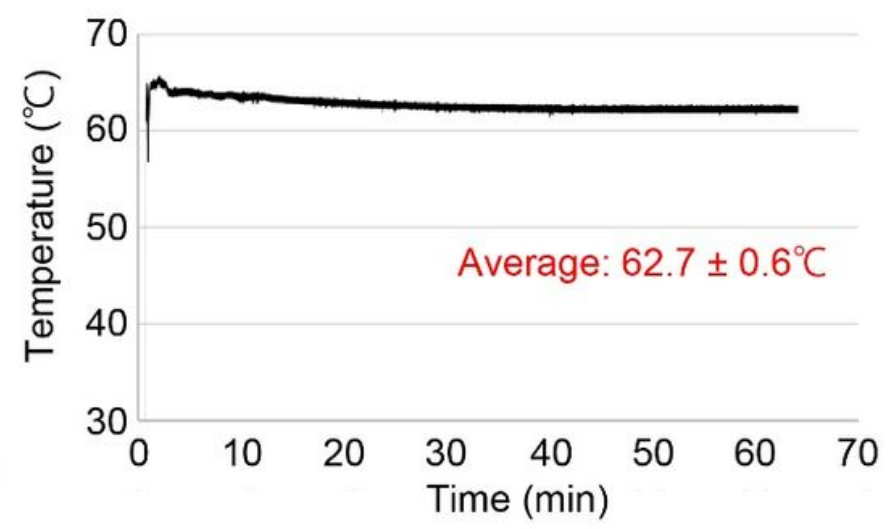

\section{Figure 3}

Temperature changes during each heating process. The graph shows the temperature in the container at each time. (a) Temperature change in the PS container during the exothermic agent reaction. (b) Temperature change of palmitic acid in the PP container during the LAMP reaction. 
(a)

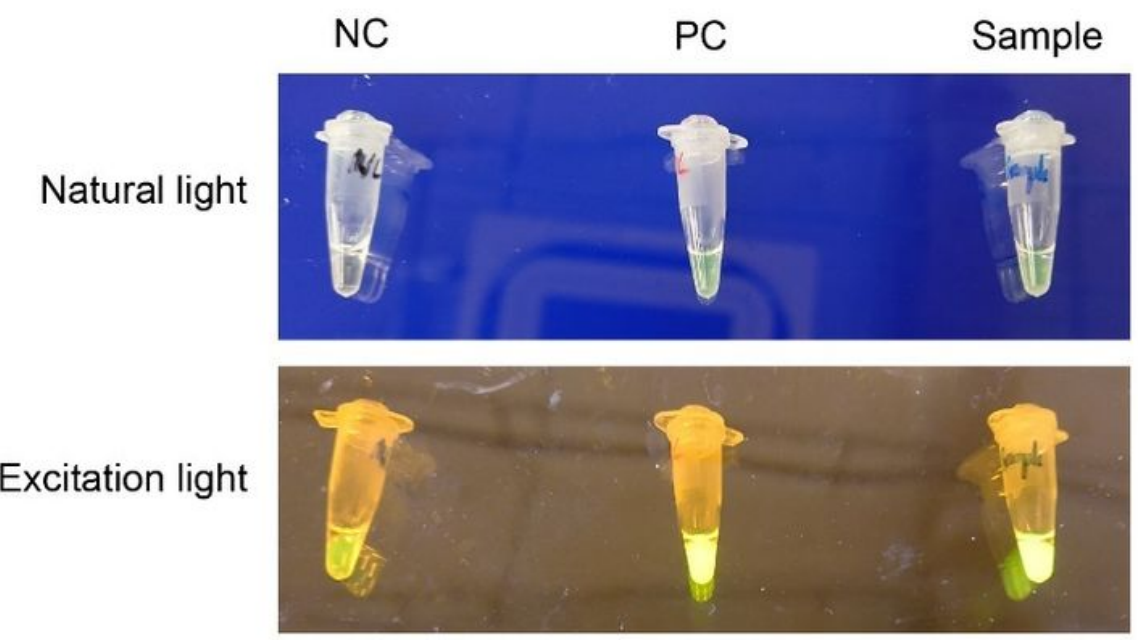

(b)

Viral concentration (copies per $\mu \mathrm{L}$ )

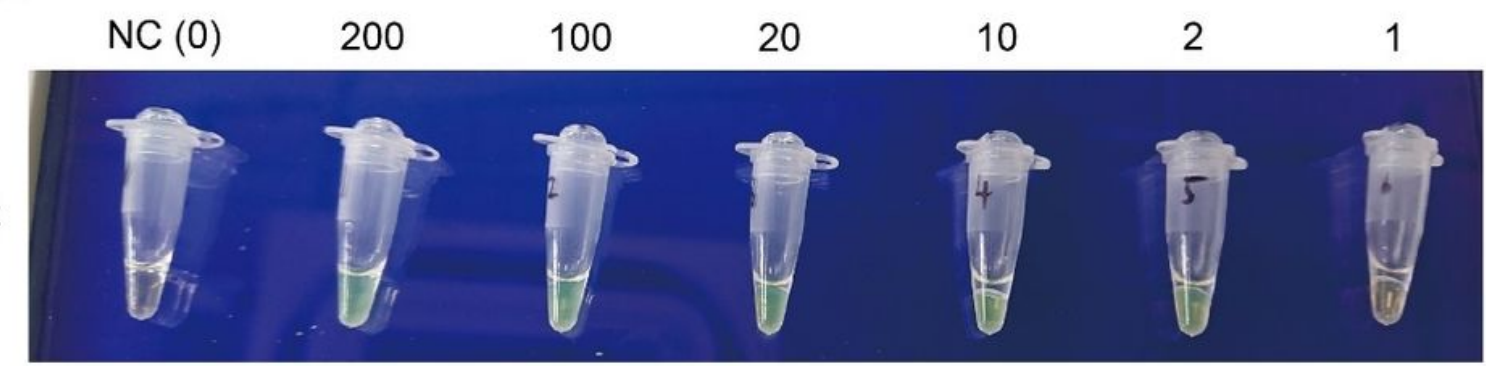

Natural light

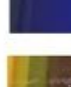

Excitation light

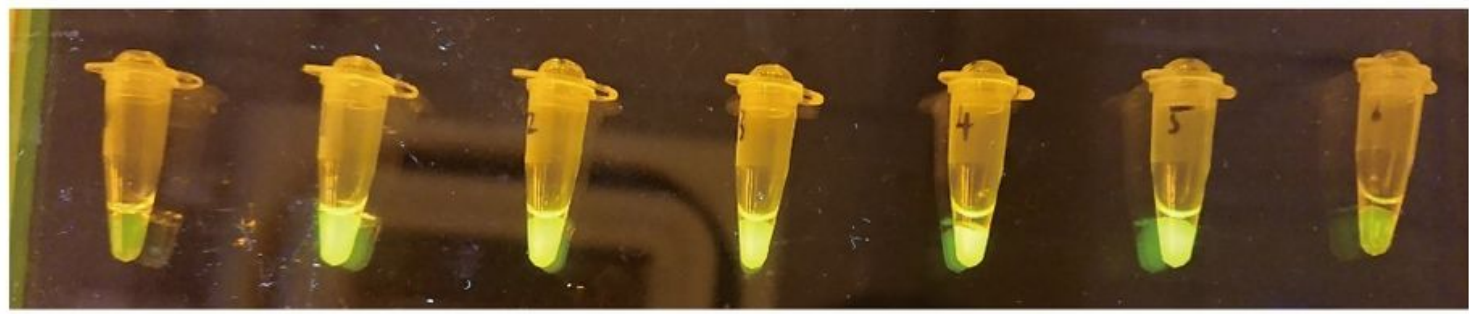

\section{Figure 4}

NEC-SD-LAMP experiment for detecting adenovirus DNA. Figures show the sample in the PCR tube after the reaction and were taken under natural or excitation light irradiation. (a) Functional comparison with a conventional system. "NC," NEC-SD-LAMP was performed using saliva samples without adenovirus DNA. "PC," SalivaDirect and LAMP assays were performed using saliva samples containing adenovirus DNA in a conventional thermal cycler. "Sample," NEC-SD-LAMP was performed using saliva samples containing adenovirus DNA. (b) Verification of the limit detection concentration of NEC-SD-LAMP. "NC," NEC-SDLAMP was performed using saliva samples without adenovirus DNA.

\section{Supplementary Files}

This is a list of supplementary files associated with this preprint. Click to download.

- SupplementaryInformation.docx 
- SupplementaryMovie.mp4

Page 17/17 